\title{
Far-Lateral Approach for Ventral and Ventrolateral Upper Cervical Meningiomas: A Case Series and Literature Review
}

\author{
Ali M. Abou-Madawi, Mohamed K. ElKazaz, Hassan A. Alshatoury, Sherif H. Ali \\ Department of Neurosurgery, Suez Canal University Hospital, Ismailia, Egypt
}

\section{Study Design: Retrospective cohort study.}

Purpose: To evaluate the safety and efficacy of the far-lateral approach in the resection of ventral and ventrolateral upper cervical meningiomas.

Overview of Literature: Upper cervical meningiomas are a common disease entity. These lesions can be surgically treated via many accesses. The far-lateral approach is a very appealing technique for these lesions.

Methods: We assessed 23 patients with a mean age of $57.3 \pm 15$ years. According to the Japanese Orthopedic Association (JOA) scale; eight patients had grade 0 , nine had grade I, and six had grade II. All patients underwent plain radiography and magnetic resonance imaging of the cervical spine. The foramen magnum was involved in 10 patients, C1-2 in seven, C2-3 in four, and C3-4 in two. All patients were operated via the far-lateral approach.

Results: Gross total resection was achieved in 21 patients. Sixteen lesions were psammomatous, five were fibroblastic, and two were meningothelial meningiomas. The mean operative duration was $135 \pm 40$ minutes, while the mean operative blood loss was $450 \pm 210 \mathrm{~mL}$, and the mean hospital stay was $4.3 \pm 2.2$ days. At the final follow-up that was conducted at $27.6 \pm 21$ months and as per the JOA score; 16 patients were classified into grade 0 and 7 into grade II. The condition of none of our patients deteriorated postoperatively. There was no significant correlation of the clinical outcome with tumor level, pathological subtype of the tumor, symptom duration, age, and sex. There was no significant correlation of tumor resection completeness with tumor level, tumor pathological subtype, or tumor topography (ventral or ventrolateral).

Conclusions: The far-lateral approach is a safe and effective access for ventral and ventrolateral cervical meningiomas. It allows direct access to tumor with no spinal cord or nerve roots traction, and thus may yield a fairly better outcome than the standard posterior approach.

Keywords: Cervical spine; Meningiomas; Ventral; Far-lateral approach; Foramen magnum

\section{Introduction}

Spinal cord tumors are common lesions that resemble
$5 \%-15 \%$ of all central nervous system tumors. Meningiomas originate from arachnoid cells, and only $10 \%$ are found extracranially [1]. Intradural extramedullary spinal

Received May 27, 2020; Revised Jun 23, 2020; Accepted Jul 4, 2020

Corresponding author: Ali M. Abou-Madawi

Department of Neurosurgery, Suez Canal University Hospital, Ring Road, Ismailia, 41511, Egypt

Tel: +20-1005110916, Fax: +20-3-4276899, E-mail: aabomadawy@med.suez.edu.eg 
tumors account for $80 \%$ of all intradural spinal tumors [1]. Spinal meningiomas represent $7.5 \%-12.7 \%$ of all meningiomas; majority of these are present in the thoracic spinal cord, and few are reported in the cervical area. Almost $27 \%$ of all whole cervical meningiomas are reported in the upper cervical spine, with only $3 \%$ being reported in the foramen magnum area $[2,3]$. Topographically, they are located either ventral or ventrolateral to the spinal cord in almost $50 \%$ of the patients.

These lesions are predominant in women, and the clinical presentation of upper cervical spine meningiomas may be nonspecific. Symptoms range from axial neck pain that is usually intense in the evening and morning to significant neurologic manifestations. Specific presentations related to neural compression include radiculopathy, myelopathy, Brown-Sequard syndrome, and complete cord transection [4].

Standard imaging modalities include contrasted magnetic resonance imaging (MRI), complementary computed tomography (CT)-scan, and plain radiography because these lesions are isointense and are rarely associated with calcifications or bone erosions [5]. Complete microsurgical excision is the gold standard treatment and can be accomplished via different accesses. As these lesions are located ventral or ventrolateral to the spinal cord, their surgical excision, without manipulation of the spinal cord and roots is challenging. Most surgeons operate these lesions via the standard posterior midline approach, while few operate via the lateral approach and rarely via the anterior midline approach [6-9]. Choosing the best access is critical and is mainly based on the spinal level, size, and topographic location of the lesion as depicted on images as well as surgeon experience and preferences.

Ventral and ventrolateral meningiomas require precise preoperative planning for a safe corridor to achieve safe and complete tumor excision [10]. Traditional posterior midline approach requires spinal cord and roots traction, and manipulation for accessing ventrally located lesions that can endanger the patient's neurology and completeness of tumor excision $[6,7,11]$. The far-lateral approach that was used in this study is a modification of the transfacetal approach (type A) of the extreme lateral craniocervical approach for ventrally located cervical lesions [12]. The lateral approach has been designed to overcome the drawbacks of the posterior midline approach [11-18]. We consider that this is the procedure of choice in our hands for these tumors. It provides safe access to these tu- mors without cervical spinal cord manipulation or effect on the vertebral and cervical muscle stability. Moreover, the positioning of patients is more convenient from the anesthetics point of view in some patients.

Here, we present our experience with the far-lateral approach to ventral or ventrolateral upper cervical and foramen magnum meningiomas. We also conducted a review of the literature and compared our results to similar previous reports and the standard posterior approach.

\section{Materials and Methods}

We conducted a retrospective descriptive clinical case cohort study with a literature review at the Neurosurgery Department, Suez Canal University Hospital, Ismailia, Egypt. We reviewed our hospital medical records from January 2007 to June 2019 for all patients operated for spinal meningiomas. We were able to trace the medical records of 113 patients who had been operated for spinal meningiomas; 49 of them were cervical patients. In this study, we enrolled only those patients who were operated via the far-lateral approach for either ventral or ventrolateral meningiomas of the upper cervical spine and foramen magnum area.

Only 27 patients met these criteria. Of these, only 23 patients had complete medical records in terms of the preoperative, operative, postoperative, and contact data; the other four were excluded because of insufficient data.

The upper cervical spine in this study involved lesions from the fourth cervical vertebra up to the foramen magnum. The foramen magnum, as described by George et al. [19], is the zone defined anteriorly by the lower onethird of clivus to the upper edge of the axis vertebral body, laterally from the jugular tubercles to the upper edge of the C2 lamina, and posteriorly from the anterior edge of the squamous occipital bone to the spinous process of the axis vertebra. Other lesions from the foramen magnum down to the lower edge of C4 lamina were also studied. Lesions below $\mathrm{C} 4$ were excluded because in these lesions, the shoulder will hinder the far-lateral approach. Moreover, we excluded redo tumors, lesions involving the posterior aspect of the Forman magnum, and upper cervical spine, or lesion originating primarily from other areas and invading our areas, as well as all other non-meningioma tumors and those treated with the standard posterior midline approach. Surgical tumor excision has been defined as total when grossly removed, subtotal when some rem- 
nant is left attached to either of the nerves or the vertebral artery, and partial when a bulky mass was left [20].

We reported all relative demographic data, clinical data, surgical technique details, pathological type of tumors, surgical complications and morbidity, and postoperative clinical and radiological outcomes. Our institutional hospital ethical review board (Research Ethics Committee of Suez Canal University Faculty of Medicine) approved the study (IRB approval no., 4122) and waived the need for informed consent from the study subjects.

Of the total 23 patients, 17 (74\%) were women and 6 (26\%) were men, with a mean age of $57.3 \pm 15$ years (range, 41-66 years). Gait disturbance was the major complaint in our patients and was reported in 15 patients (65\%), while radicular pain was reported only in $5(21.7 \%)$, and urinary precipitancy was reported in 2 (8.7\%). The mean preoperative duration of the symptoms was $13.4 \pm 10$ months (range, 5-27 months). As per the 17-points Japanese Orthopedic Association (JOA) scale [21], eight patients $(34.8 \%)$ were classified into grade 0 (16-17), 9 (39\%) into grade I (12-15), and 6 (26\%) into grade II (8-13) (Fig. $1)$. Five patients had hypertension, four had diabetes mellitus, and one had rheumatoid arthritis.

Plain radiography was performed for all patients in the anteroposterior and lateral views; T-1 and T-2 weighted images MRI of the cervical spine with gadolinium enhancement in the sagittal, coronal, and axial views; and six patients were submitted to multiple sclerosis-CTscan. Seven patients (30.4\%) experienced mainly ventral meningiomas to the spinal cord, and 16 (69.6\%) had ventrolateral meningiomas. The foramen magnum was involved in 10 patients, C1-2 segment in seven, C2-3 segment in four, and C3-4 segment in two (Fig. 2). Based on

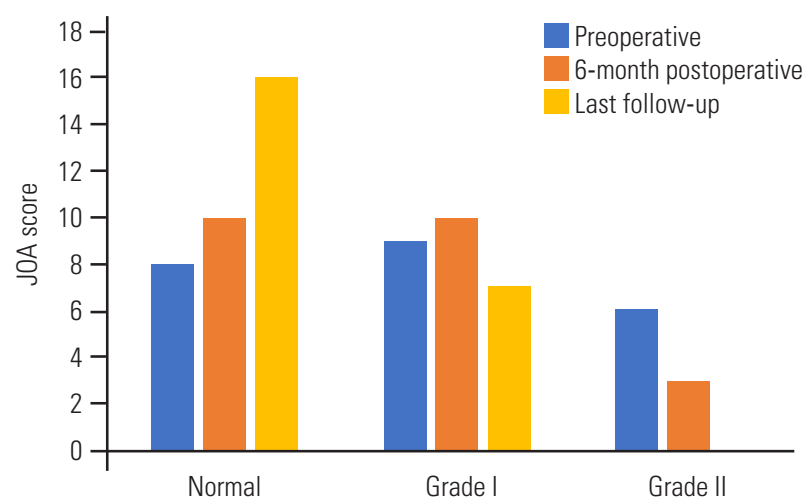

Fig. 1. Preoperative, 6-month postoperative, and last follow-up JOA score of study patients. JOA, Japanese Orthopedic Association. the topographic location of the meningiomas, 15 patients were approached from the left side, and only eight were operated from the right side. All the meningiomas were considered large because their cross-sectional area exceeded $50 \%$ of the spinal canal as per the measurement on MRI axial images.

\section{Operative technique}

The patient was placed in the lateral decubitus position under general anesthesia with the head fixed in a Mayfield head clamp. The head was fixed in the dead lateral position with some flexion and contralateral tilt. An incision was marked as per the target segment either straight or lazy "S" one finger-breadth behind the mastoid process. Dissection started between the sternomastoid muscle anteriorly and the trapezius muscle posteriorly and down in the facial plan between the scaleni muscles anteriorly and levator scapulae posteriorly, continuing deeply with finger palpations targeting the tip of the atlas transverse process. In the lower pathology, the fingertip was directed to the lateral mass of the target cervical segment.

C1 transverse process was palpated just inferior to the mastoid tip; thereafter, the muscles attachments to it were resected. The ipsilateral lamina of the cervical spine was palpated, and a Cobb muscle elevator was used to remove the deep cervical muscle attachments subperiosteally and gently from the lamina to protect the vertebral artery from injury [22]. Exposure of the hemi-laminae according to the target level was conducted. Using high-speed drill and Kerrison rongeur, laminotomy was performed and extended from the fact joints anteriorly to the base of the corresponding spinous process posteriorly, using the retro-facet approach. In a similar manner, and in the foramen magnum meningiomas, a suboccipital craniotomy could be performed. After full dural exposure, a vertical dural incision as per the target level was performed, and

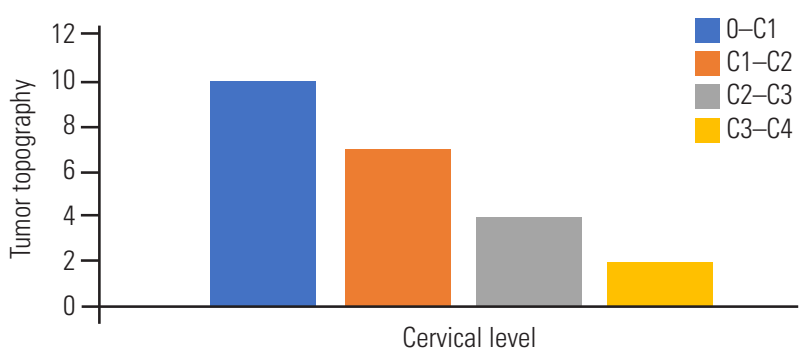

Fig. 2. Tumor level reported in this study. 
dural edges were stitched to the ipsilateral muscles. Dentate ligaments were sharply incised to allow tumor access and relax the spinal cord.

Tumor debulking was performed using a combination of thermal bipolar coagulation, rongeurs, curettes, and ultrasonic aspirator. In the case of some meningiomas, the lesion was easily suckable through the suction appa- ratus. Internal tumor cavitation of the mass allows tumor mobilization with limited cord traction. Neurophysiological monitoring, when available, was essential for safe complete mass removal and prevention of neurological deficit [4]. This facility was available only in the final three patients. If the tumor was not big enough, we used bipolar cauterization in the plan between the dura and tumor;
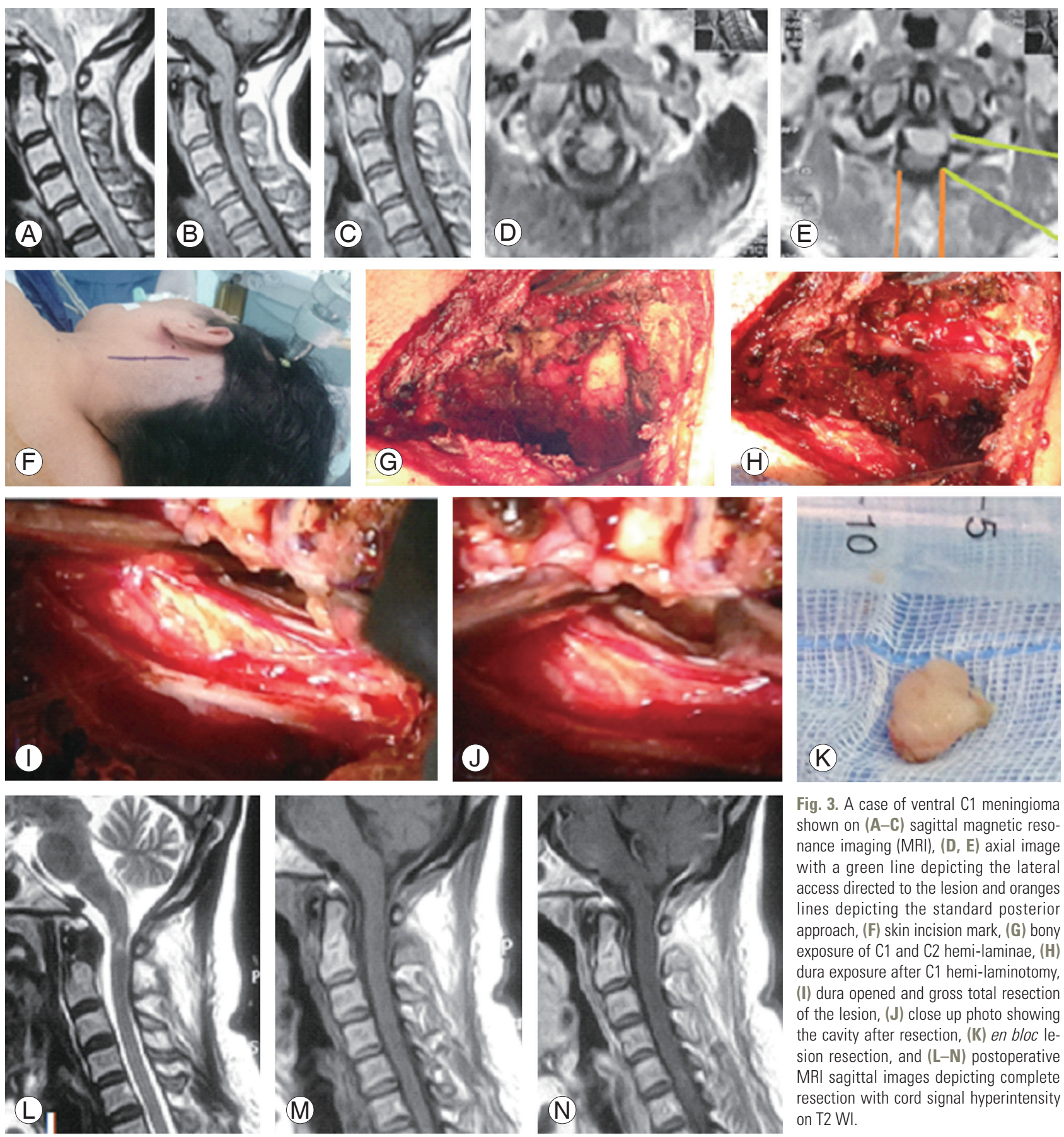

Fig. 3. A case of ventral C1 meningioma shown on $(\mathbf{A}-\mathbf{C})$ sagittal magnetic resonance imaging (MRI), (D, E) axial image with a green line depicting the lateral access directed to the lesion and oranges lines depicting the standard posterior approach, (F) skin incision mark, (G) bony exposure of $\mathrm{C} 1$ and $\mathrm{C} 2$ hemi-laminae, $(\mathrm{H})$ dura exposure after $\mathrm{C} 1$ hemi-laminotomy, (I) dura opened and gross total resection of the lesion, (J) close up photo showing the cavity after resection, (K) en bloc lesion resection, and (L-N) postoperative MRI sagittal images depicting complete resection with cord signal hyperintensity on T2 WI. 
thus, the tumor was de-vascularized and removed it as one piece (Figs. 3, 4). The dural base needed to be cleaned and/or coagulated with bipolar at the end of each procedure. On two occasions, a small piece of the tumor was attached to the ipsilateral intradural vertebral artery and cauterized using bipolar and left in situ (Fig. 5).

Adequate hemostasis was ensured, water-tight closure of the dura was performed, and an epidural closed drainage system was inserted; its track is designed through muscle and skin away from the deep facial suture line. The wound was meticulously closed in layers obliterating any dead space, and sterile drape was applied. The drain was removed after 24 hours if there was no cerebrospinal fluid (CSF) leakage in the drain. If CSF came out through the drain, it was left in situ until the CSF stopped or left for a week and then removed. Parenteral third generation cephalosporins were continued for 3 days perioperatively. Patients were discharged from the hospital after drain removal and scheduled for outpatient clinic visits.

All the patients were followed up routinely and regu- larly at the outpatient clinic for 12 months. Postoperative MRI with gadolinium enhancement was performed for all patients to ensure completeness of tumor excision.

\section{Statistical analyses}

The IBM SPSS statistical software program ver. 25.0 (IBM Corp., Armonk, NY, USA) was used for statistical analysis of the study data. The outcome descriptive data are presented as mean, standard deviation, and range values. Kolmogorov-Smirnov test was performed to prove data to be parametric. For comparison of pre-post data, paired $t$-test was used. For comparison between the data groups, independent samples $t$-test was used. A chi-square test was used to compare the proportions and prove associations between variables. Univariate and multivariate testing were used to check the associations between different variables. Spearman correlation coefficients were calculated among the variables. A $p$-value $<0.05$ was considered to indicate statistical significance.
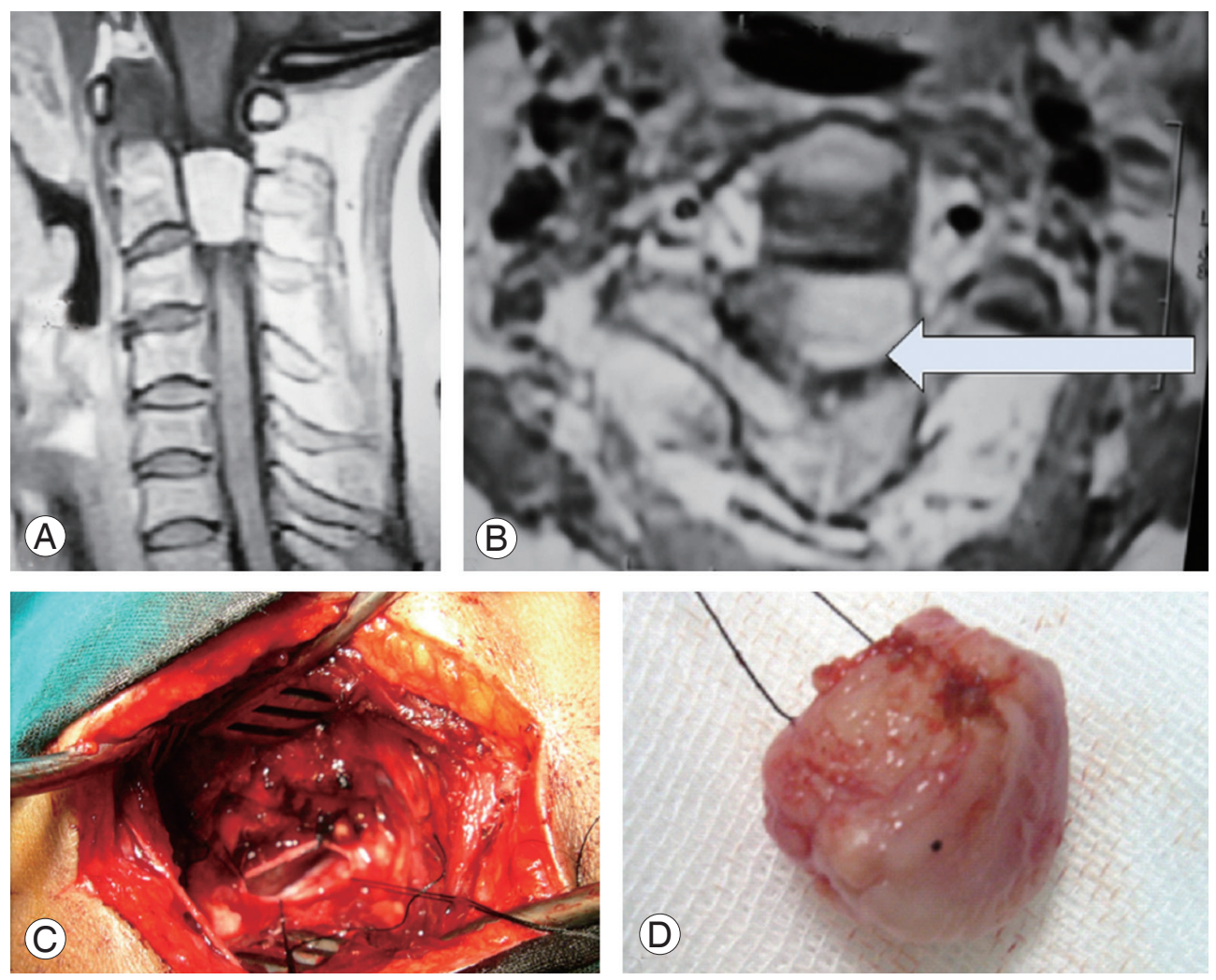

Fig. 4. A case of C2 left ventrolateral meningioma. (A) Preoperative post-gadolinium enhanced sagittal T1 WI magnetic resonance imaging (MRI) cervical spine. (B) Axial T1 WI MRI depicting the mass (arrow). (C) Operative photo showing surgical access following en bloc tumor resection and depicting contralateral dura. (D) En bloc resected meningioma. 


\section{Results}

The far-lateral approach was used in 23 ventral and ventrolateral upper cervical meningiomas. In all our patients, the lateral approach was conducted smoothly and pro- vided excellent exposure and good access that enabled effective tumor resection. All the patients had soft operative and postoperative course without significant adverse events. Operatively documented total tumor removal was achieved in 21 patients and subtotal in two where a small
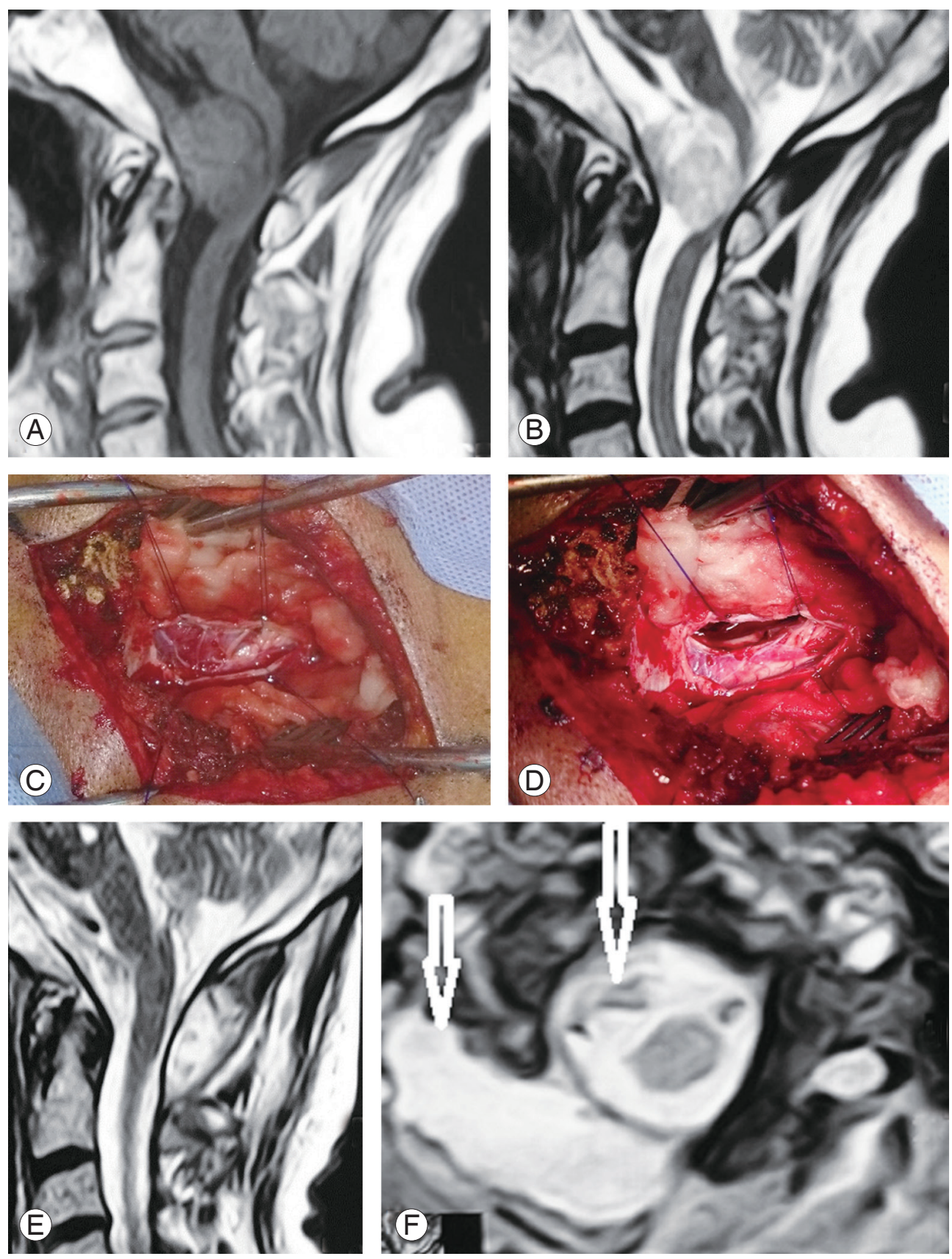

Fig. 5. A case of foramen magnum large meningioma squeezing the upper cervical cord and lower medulla. (A) T1 WI sagittal MRI. (B) T2 WI magnetic resonance imaging (MRI) depicting the lesion. (C) Operative photo after right side C1 laminotomy and suboccipital craniotomy followed by dural opening and tumor exposure. (D) After piece meal, gross total resection and tiny piece were attached to the vertebral artery and coagulated in situ. (E) Sagittal T1 WI MRI showing the residual tiny tumor. (F) Axial T2 WI MRI showing the tiny residual tumor, with complete cord decompression and residual pseudomeningocele (arrows). 
Table 1. Perioperative parameters $(n=23)$

\begin{tabular}{lc} 
Characteristic & Value \\
\hline Operative time (min) & $135 \pm 40(110-380)$ \\
\hline Blood loss (mL) & $450 \pm 210(200-850)$ \\
\hline Hospital stay (day) & $4.3 \pm 2.2(2-7)$ \\
\hline Gross total excision & 21 \\
\hline Subtotal excision & 2 \\
Cerebrospinal fluid drain collection & 3 \\
\hline Follow-up (mo) & $27.6 \pm 21(12-60)$ \\
\hline
\end{tabular}

Values are presented as mean \pm standard deviation (range) or number.

tumor portion was left attached to the intradural vertebral artery and was cauterized in situ with bipolar. This was documented in the postoperative MRI that was performed for all patients (Fig. 5). The mean operative time was $135 \pm 40$ minutes (range, 110-380 minutes, while the mean operative blood loss was $450 \pm 210 \mathrm{~mL}$ (range, 200-850 $\mathrm{mL}$ ), and the mean hospital stay was $4.3 \pm 2.2$ days (range, 2-7 days) (Table 1 ).

Histopathological results revealed that psammomatous meningiomas were reported in 16 patients (69.6\%), fibroblastic meningiomas in five patients (21.7\%), and meningothelial meningiomas in two patients (8.7\%).

At the final follow-up that was conducted at $27.6 \pm 21$ months (range, 12-60 months) and according to the JOA score; 16 patients were classified into grade 0 (normal, 16-17) and seven into grade II (12-15) (Fig. 1). Root pain also improved in all five patients with preoperative radiculopathy and urinary precipitancy, while gait disturbance improved remarkably in eight patients (53\%) and moderately in seven patients (47\%). One patient showed asymptomatic local tumor recurrence 3 years after the surgery on routine MRI imaging and refused further surgery, while preferring to undergo follow-up.

Univariate and multivariate analyses showed that there were no significant associations of clinical outcome with tumor severity, pathological subtype of the tumor, duration of symptoms, age, and sex. Moreover, there were no significant associations of tumor resection completeness with the tumor level, tumor pathological subtype, or tumor topography (ventral or ventrolateral). Spearman correlation showed no statistically significant correlations among the study variables.

In four patients, the drain was left in situ because of CSF leakage into the drain for 5-7 days; thereafter, the drain was removed. One patient experienced superficial wound infection that was treated with frequent dressing and parental antibiotics for 1 week. Another patient experienced DVT that was treated with anticoagulants and followed up with our hospital vascular outpatient clinic as well. There was no reported neurological deterioration or other reported morbidity or mortality.

\section{Discussion}

Ventral and ventrolateral meningiomas of the upper cervical spine and foramen magnum pose a challenge to surgeons treating these lesions. The surgical removal of these lesions through the standard posterior midline approach involves a high risk of morbidities, and total resection is not possible in certain patients [9]. The far-lateral approach provides an alternate safe access that allows adequate exposure and gross total resection in these ventral and ventrolateral meningiomas of the upper cervical cord and brain stem [19].

Upper cervical meningiomas and foramen magnum are common neoplasms. Foramen magnum meningiomas represent 3\% [3], high-cervical lesions represent 27\% $(n=9)$, and low-cervico-thoracic lesions represent $73 \%$ $(\mathrm{n}=24)$ of all spinal meningioma cases [2].

In 1988, George et al. [23] introduced and described the far-lateral approach for tumors anterior to the foramen magnum, a technique similar to that described by Heros [24] for the treatment of vertebral and vertebrobasilar lesions. The far-lateral approach and its variations have become a standard technique for many surgeons dealing with the foramen magnum and upper cervical lesions [12].

This retrospective clinical cohort study reports on 23 patients with upper cervical meningiomas from $\mathrm{C} 4$ up to the foramen magnum. The mean patient age was 57 years, with $74 \%$ of the patients being women. All patients were operated using the far-lateral approach. The far-lateral approach is a unique and fairly recent approach that is not commonly used in comparison to the standard posterior midline approach. It has been assumed that this technique is safe and effective, especially for ventral and ventrolateral cervical meningiomas. Our experience and results in these patients advocate the safety and efficacy of this technique.

\section{Cervical meningiomas characteristics}

In this series, the mean patient age was $57 \pm 15$ years 
(range, 41-66 years). Other series have reported similar figures with mean ages of 63, 60, and 55.6 years, respectively $[2,25,26]$. In our series, $74 \%$ of the patients were women, and $26 \%$ were men. Similar sex distribution has been reported previously $[2,25,26]$. The mean duration of preoperative symptoms in this series was $13 \pm 10$ months, and the mean duration of symptoms was 10.3 months in a previous trial [26].

\section{Posterior versus anterior technique}

Since several decades, the standard posterior midline approach has been practiced with reasonable safety and good clinical outcomes. Although posterior or posterolateral meningiomas can be safely and effectively resected using the standard posterior midline approach, the optimal approach for ventral or ventrolateral meningiomas remains controversial. Resection of these meningiomas could result in significant spinal cord or brain stem retraction, inadequate access and exposure, high rate of incomplete resection, and high morbidity $[19,26]$. Many authors have reported that patients operated via the standard posterior midline approaches showed worse clinical outcomes [14,27]. Goel et al. [28] reported what was contradicting that, when he reported gross total removal of 14 out of 17 ventral or ventrolateral meningiomas with similar results to other authors.

\section{Some technical details}

A key point of the far-lateral approach is the manipulation of the vertebral artery. Our approach is a modification of the trans-facetal variation proposed by Salas et al. [12], while we used the retro-facet approach. Our surgical corridor or access for the intradural lesion was a laminotomy between the fact joints anteriorly and the base of the corresponding spinous processes posteriorly. This approach was designed only for intradural tumors; where, in these lesions, there is no need to drill the facets or the condyles. In extradural lesions with bony involvement, it is mandatory to mobilize the vertebral artery and hinge it anteriorly to achieve complete tumor resection.

In our series, tumor topography and relation to the spinal cord were the major determinants of the surgical access; $30.4 \%(n=7)$ were ventral, and $69.6 \%(n=16)$ were ventrolateral. Slin'ko and Al-Qashqish [25] reported 24\% ventral lesions and $76 \%$ ventrolateral; they used different approaches in their series, including dorsal, dorsolateral, and ventrolateral, depending on the tumor severity and topography. They also reported that the clinical outcome in patients with ventral lesions was worse than in those with ventrolateral lesions. Similarly, Schaller [2] reported ventral tumor position in six patients $(18 \%)$, ventrolateral in 19 (58\%), and posterior in 8 (24\%).

According to our technique, and in agreement with Joaquim et al. [6] who proposed a technique of cutting the dentate ligaments and mobilizing the spinal cord to protect against neurologic injury and reported no neurologic deterioration in their technique demonstration. Dentate ligaments cutting relaxes the spinal cord, facilitates spinal cord and spinal roots manipulation, and prevents cord traction and root tension during tumor resection.

The histopathological subtype plays an important role in the ease of performing the procedure because the meningothelial lesion was easily suckable and could be removed easily with the suction nozzle, while the psammomatous lesion was tough, hard, and fibrotic that necessitated the use of ultrasonic aspiration for safe and complete tumor resection.

If we look critically at the MRI images of these ventral and ventrolateral meningiomas, we can see that the axis of vision of the surgeon as well as the surgical access and the working corridor for these tumors are more direct in the lateral approach (Figs. 6, 7; white and green lines). Moreover, in the posterior approach, the surgeon would work indirectly on a big part of the tumor (Figs. 6, 7; yellow and orange lines). These images clearly demonstrate the advantages of the visual axis and surgical corridor of the lateral approach that would be disadvantageous with the posterior approach. This significantly affects the safety of this procedure and the ability to remove such ventrally located lesions entirely.

\section{Histopathology}

Histopathological results revealed that psammomatous meningiomas were reported in 16 patients $(69.6 \%)$, fibroblastic meningiomas in five patients $(21.7 \%)$, and meningothelial meningiomas in two patients (8.7\%). Similar reports $(66 \%, 22 \%$, and $11 \%$, respectively) have been documented previously [2], while other studies have reported contradictory findings $[3,10,19,20,25,26]$, which reported the meningothelial subtype as the most common in $49 \%$, $90 \%, 56 \%, 39 \%$, and $55 \%$, respectively. The analysis of our 

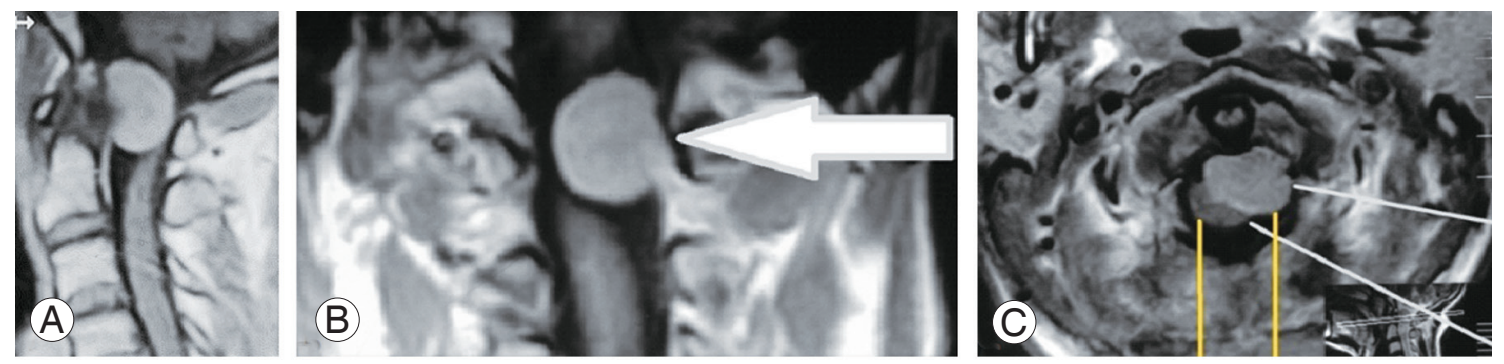

Fig. 6. A case of $\mathrm{C} 1$ meningioma. (A) Post-gadolinium enhanced sagittal T1 WI magnetic resonance imaging (MRI). (B) Coronal T1 WI MRI showing right ventrolateral meningioma (big white arrow). (C) Axial T1 WI post-gadolinium enhanced MRI showing ventrolateral right lesion with the white lines depicting the far-lateral access where we operated; the yellow lines depicting the standard posterior approach that we did not prefer.
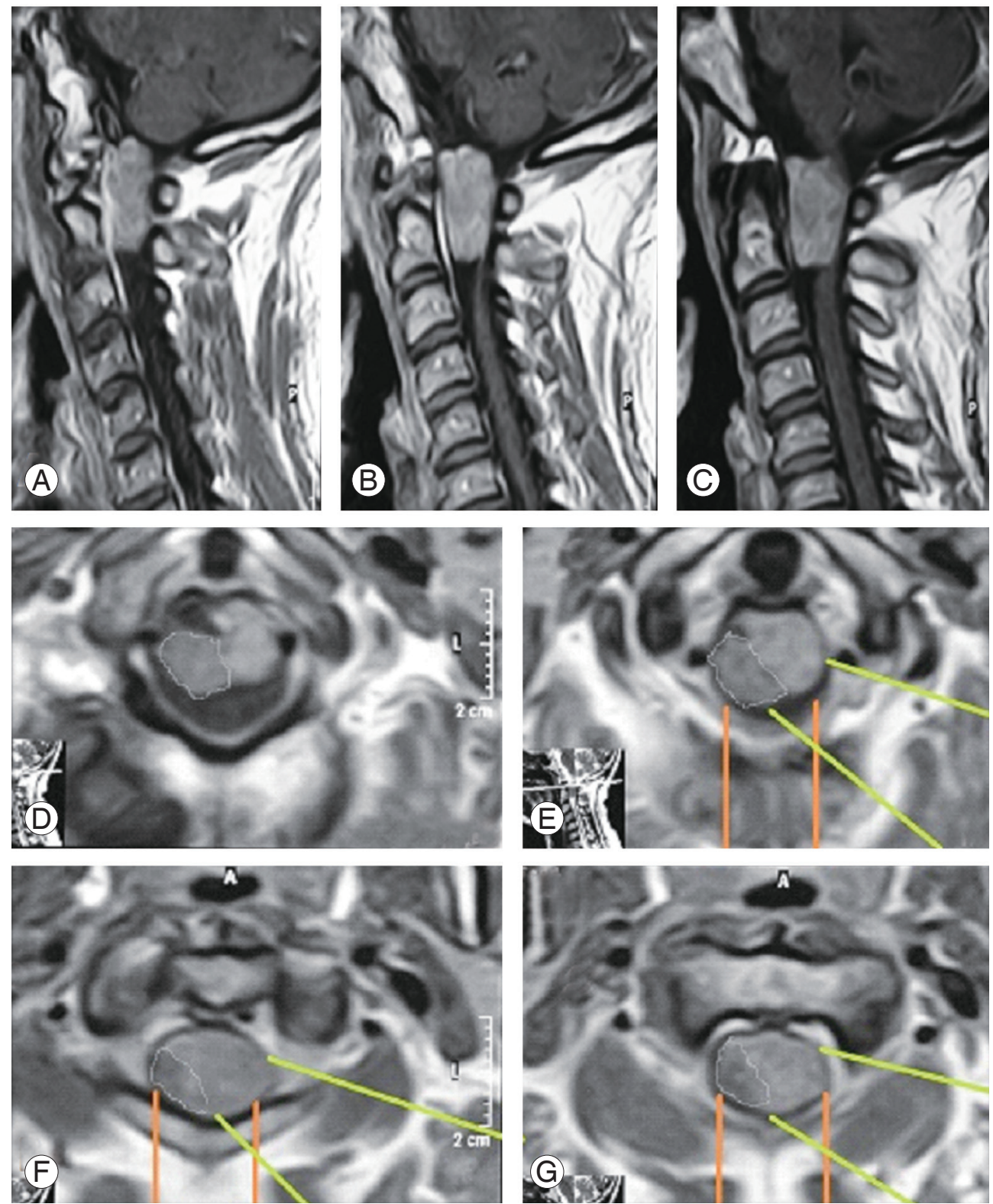

Fig. 7. A case of large C1/2 meningioma. (A-C) Post-gadolinium enhanced sagittal TI WI magnetic resonance imaging (MRI) images. (D-G) Serial axial T1 WI post-gadolinium enhanced MRI depicting the topography of the lesion with the green lines demonstrating the far-lateral access where we operated, and the orange lines demonstrating the standard posterior approach. 
reported data and that reported by others has shown that the histopathological subtypes did not influence the clinical or radiological outcomes [3,26,29].

\section{Clinical and radiological outcomes}

According to the JOA score, $70 \%$ of our patients recovered completely, $30 \%$ showed reduction in their preoperative symptoms, and none of them showed worsening of the condition. Flores et al. [26] reported full recovery of preoperative neurology in $73 \%$ of his patients. Slin'ko and Al-Qashqish [25] conducted a 39-month follow-up study and reported full recovery in 70 patients (50\%), improvement in 53 patients (38\%), no change in 10 patients (7\%), and neurological deterioration in seven patients (5\%). In his series, Schaller [2] reported that preoperative neurological deficits resolved in $79 \%(\mathrm{n}=26)$ and deteriorated in $21 \%(n=7)$. Further, he added that all the latter had meningiomas of the psammomatous type. He stressed that surgery of spinal psammomatous meningiomas was associated with a less favorable outcome than surgery of other pathological subtypes [2]. In contrast, Arnautovic et al. [3] reported that the pathological subtype did not influence the clinical outcome; however, the meningothelial subtype was associated with most of the gross total removal cases.

Some studies have reported good prognostic outcomes with the following factors: posterior or lateral tumor position in the spinal canal, location below $\mathrm{C} 4$, age $<60$ years, duration of preoperative symptoms, total tumor resection, adequate access and exposure, no cord traction, and microsurgical technique [2,25]. One other important factor was that the general outcome and recovery was associated with the date of each reported series; thus, more recent the series, better the patient outcome. This reflects the improvement in facilities as well as the experience of the involved team.

Gross total removal was achieved in $91 \%$ of our patients; this was achieved in $85.7 \%, 67 \%$ by others $[3,26]$. Total tumor resection was achieved via different posterior approaches in $74 \%$ of the patients in an earlier trial [25]. We found no impact of the histological subtypes on tumor resection or clinical outcome, as reported by others [26,29].

A literature review showed that the general outcome was better with the lateral approach as compared to that with the standard posterior midline approach $[3,6,7,25,30]$. This difference was related to the group of patients with ventral and ventrolateral meningiomas who have been treated with the posterior approach.

\section{Morbidity}

We had no case of postoperative neurological deterioration in this series; however, previous studies have reported a prevalence of $7 \%$ and $3 \%[25,26]$. The first series used different surgical approaches; this explains the increased morbidity with posterior approaches [25].

We reported four patients with continuous CSF collection into the drain who responded with time and conservative measures. This was reported in another lateral approach as CSF collection in one patient out of 10 responded to conservative measures [17], CSF leakage in three patients out of 30 responded to temporary external drainage [26], CSF leak in two of six with controlled well [27], they attributed this leakage to excessive dural cauterization to control bleeding during the procedure. Meanwhile, one study that employed the posterior approach reported that only one of 18 patients had CSF leakage [7].

We reported only one case with recurrence at postoperative 3 years. Studies that have used the lateral approaches reported no recurrence at the 5-year follow-up [26,30]. In our series, we did not report any postoperative lower cranial nerve palsy in any patient. This was reported in many other studies, especially those that reported on lesions at the foramen magnum $[3,9,26]$.

\section{Adjuvant therapy}

Fortunately, all our meningiomas were World Health Organization grade I, and only in two patients, a very small piece of the tumor was left attached to the vertebral artery and cauterized in situ. Thus, there was no need for adjuvant therapy. Fractionated stereotactic radiosurgery (CyberKnife; Accuray Inc., Sunnyvale, CA, USA) adjuvant therapy was used by Flores et al. [26] on one occasion with successful local tumor control. Adjuvant stereotactic radiosurgery should be considered in patients with residual or growing residual tumors or if reoperation carries a high rate of morbidity.

\section{Study limitations}

It would be more useful and informative to perform more studies with a larger sample size and longer follow-up 
duration to confirm the recurrence rate. Therefore, we believe that a multi-center study would be more interesting in this respect.

\section{Conclusions}

The far-lateral approach is a safe and effective access for ventral and ventrolateral cervical meningiomas. It allows direct access to tumors with no spinal cord or nerve roots traction, and thus yields a fairly better outcome than the standard posterior approach.

\section{Conflict of Interest}

No potential conflict of interest relevant to this article was reported.

\section{ORCID}

Ali M. Abou-Madawi: https://orcid.org/0000-0003-0581-6458; Mohamed K. ElKazaz: https://orcid.org/0000000272905744; Hassan A. Alshatoury: https://orcid.org/0000000173856510; Sherif H. Ali: https://orcid.org/0000000335737283

\section{Author Contributions}

Ali M. Abou-Madawi: operating upon cases and writing manuscript; Mohamed K. ElKazaz: data collection and writing manuscript; Hassan A. Alshatoury: operating upon cases and writing manuscript; and Sherif H. Ali: data collection and writing manuscript.

\section{References}

1. Haegelen C, Morandi X, Riffaud L, Amlashi SF, Leray E, Brassier G. Results of spinal meningioma surgery in patients with severe preoperative neurological deficits. Eur Spine J 2005;14:440-4.

2. Schaller B. Spinal meningioma: relationship between histological subtypes and surgical outcome? J Neurooncol 2005;75:157-61.

3. Arnautovic KI, Al-Mefty O, Husain M. Ventral foramen magnum meninigiomas. J Neurosurg 2000;92(1 Suppl):71-80.

4. Sandalcioglu IE, Hunold A, Muller O, Bassiouni H, Stolke D, Asgari S. Spinal meningiomas: critical review of 131 surgically treated patients. Eur Spine J
2008;17:1035-41.

5. Gezen F, Kahraman S, Canakci Z, Beduk A. Review of 36 cases of spinal cord meningioma. Spine (Phila Pa 1976) 2000;25:727-31.

6. Joaquim AF, Almeida JP, Dos Santos MJ, Ghizoni E, de Oliveira E, Tedeschi H. Surgical management of intradural extramedullary tumors located anteriorly to the spinal cord. J Clin Neurosci 2012;19:1150-3.

7. Kim CH, Chung CK. Surgical outcome of a posterior approach for large ventral intradural extramedullary spinal cord tumors. Spine (Phila Pa 1976) 2011;36:E531-7.

8. Barami K, Dagnew E. Endoscope-assisted posterior approach for the resection of ventral intradural spinal cord tumors: report of two cases. Minim Invasive Neurosurg 2007;50:370-3.

9. Pai SB, Raghuram G, Keshav GC, Rodrigues E. Farlateral transcondylar approach to anterior foramen magnum lesions: our experience. Asian J Neurosurg 2018;13:651-5.

10. Aboul-Enein HA, Khidr WM, Abdeen KM, Madawi AA. Surgical management of ventrally based lower cervical (subaxial) meningiomas through the lateral approach: report on 16 cases. Clin Neurol Neurosurg 2015;139:152-8.

11. Lanzino G, Paolini S, Spetzler RF. Far-lateral approach to the craniocervical junction. Neurosurgery 2005;57(4 Suppl):367-71.

12. Salas E, Sekhar LN, Ziyal IM, Caputy AJ, Wright DC. Variations of the extreme-lateral craniocervical approach: anatomical study and clinical analysis of 69 patients. J Neurosurg 1999;90(2 Suppl):206-19.

13. Kratimenos GP, Crockard HA. The far lateral approach for ventrally placed foramen magnum and upper cervical spine tumours. Br J Neurosurg 1993;7:129-40.

14. Sen C, Shrivastava R, Anwar S, Triana A. Lateral transcondylar approach for tumors at the anterior aspect of the craniovertebral junction. Neurosurgery 2010;66(3 Suppl):104-12.

15. Margalit NS, Lesser JB, Singer M, Sen C. Lateral approach to anterolateral tumors at the foramen magnum: factors determining surgical procedure. Neurosurgery 2005;56(2 Suppl):324-36.

16. Angevine PD, Kellner C, Haque RM, McCormick PC. Surgical management of ventral intradural spinal lesions. J Neurosurg Spine 2011;15:28-37. 
17. Ayoub B. The far lateral approach for intra-dural anteriorly situated tumours at the craniovertebral junction. Turk Neurosurg 2011;21:494-8.

18. Liu JK, Rao G, Schmidt MH, Couldwell WT. Far lateral transcondylar transtubercular approach to lesions of the ventral foramen magnum and craniovertebral junction. Contemp Neurosurg 2007;29:1-7.

19. George B, Lot G, Boissonnet H. Meningioma of the foramen magnum: a series of 40 cases. Surg Neurol 1997;47:371-9.

20. Moscovici S, Umansky F, Spektor S. "Lazy" far-lateral approach to the anterior foramen magnum and lower clivus. Neurosurg Focus 2015;38:E14.

21. Azimi P, Mohammadi HR, Montazeri A. An outcome measure of functionality and pain in patients with lumbar disc herniation: a validation study of the Japanese Orthopedic Association (JOA) score. J Orthop Sci 2012;17:341-5.

22. Naito K, Yamagata T, Kawahara S, Ohata K, Takami T. High cervical lateral approach to safely remove the cystic retro-odontoid pseudotumor: technical note. Neurol Med Chir (Tokyo) 2019;59:392-7.

23. George B, Dematons C, Cophignon J. Lateral approach to the anterior portion of the foramen magnum: application to surgical removal of 14 benign tumors: technical note. Surg Neurol 1988;29:484-90.
24. Heros RC. Lateral suboccipital approach for vertebral and vertebrobasilar artery lesions. J Neurosurg 1986;64:559-62.

25. Slin'ko EI, Al-Qashqish II. Intradural ventral and ventrolateral tumors of the spinal cord: surgical treatment and results. Neurosurg Focus 2004;17:ECP2.

26. Flores BC, Boudreaux BP, Klinger DR, Mickey BE, Barnett SL. The far-lateral approach for foramen magnum meningiomas. Neurosurg Focus 2013;35:E12.

27. Sen CN, Sekhar LN. An extreme lateral approach to intradural lesions of the cervical spine and foramen magnum. Neurosurgery 1990;27:197-204.

28. Goel A, Desai K, Muzumdar D. Surgery on anterior foramen magnum meningiomas using a conventional posterior suboccipital approach: a report on an experience with 17 cases. Neurosurgery 2001;49:102-7.

29. Bassiouni H, Ntoukas V, Asgari S, Sandalcioglu EI, Stolke D, Seifert V. Foramen magnum meningiomas: clinical outcome after microsurgical resection via a posterolateral suboccipital retrocondylar approach. Neurosurgery 2006;59:1177-87.

30. Komotar RJ, Zacharia BE, McGovern RA, Sisti MB, Bruce JN, D’Ambrosio AL. Approaches to anterior and anterolateral foramen magnum lesions: a critical review. J Craniovertebr Junction Spine 2010;1:86-99. 